\title{
ABSOLUTELY PURE MODULES
}

\author{
CHARLES MEGIBBEN ${ }^{1}$
}

Abstract. A module $A$ is shown to be absolutely pure if and only if every finite consistent system of linear equations over $A$ has a solution in $A$. Noetherian, semihereditary, regular and Prüfer rings are characterized according to properties of absolutely pure modules over these rings. For example, $R$ is Noetherian if and only if every absolutely pure $R$-module is injective and semihereditary if and only if the class of absolutely pure $R$-modules is closed under homomorphic images. If $R$ is a Prüfer domain, then the absolutely pure $R$-modules are the divisible modules and $\operatorname{Ext}_{R}^{1}(M, A)=0$ whenever $A$ is divisible and $M$ is a countably generated torsionfree $R$-module.

Throughout $R$ will denote an associative ring with identity and all modules are unital. An $R$-module, without further qualification, will always be a left $R$-module. Similarly, Noetherian and semihereditary will mean left-Noetherian and left semihereditary, respectively. A submodule $A$ of the $R$-module $B$ is said to be a pure submodule if for all right $R$-modules $M$ the induced map $M \otimes_{R} A \rightarrow M \otimes_{R} B$ is monic. An equivalent formulation of purity more useful for our purposes is that the induced map $\operatorname{Hom}_{R}(M, B) \rightarrow \operatorname{Hom}_{R}(M, B / A)$ be surjective for all finitelypresented $R$-modules $M-M$ is finitely presented if it is the quotient of a finitely generated free $R$-module by a finitely generated submodule. Maddox [4] has called a module absolutely pure if it is pure in every module containing it as a submodule. As we shall see, an equally appropriate appellation for such modules would be finitely injective. Now if $A$ is pure in $B$ and if $C$ is a submodule of $B$ containing $A$, then it is easy to see that $A$ is pure in $C$. Therefore $A$ is absolutely pure if and only if $A$ is pure in every injective module containing $A$ and hence if and only if $A$ is pure in its injective envelope.

Proposition 1. An R-module $A$ is absolutely pure if and only if $\operatorname{Ext}_{R}^{1}(M, A)=0$ for all finitely presented $R$-modules $M$.

Proof. Let $E$ be the injective envelope of $A$. We then have the exact sequence

Received by the editors November 10, 1969.

AMS 1969 subject classifications. Primary 1690; Secondary 1640, 1625.

Key words and phrases. Pure submodule, absolutely pure, injective module, Noetherian ring, semihereditary ring, regular ring, Prüfer domain, divisible module, torsion-free module.

1 This research was supported by National Science Foundation Grant GP-11804. 


$$
\operatorname{Hom}_{R}(M, E) \stackrel{\theta}{\rightarrow} \operatorname{Hom}_{R}(M, E / A) \rightarrow \operatorname{Ext}_{R}^{1}(M, A) \rightarrow 0 .
$$

Obviously $\theta$ is surjective if and only if $\operatorname{Ext}_{R}^{1}(M, A)=0$.

Corollary 1. If $A$ is an absolutely pure $R$-module, then $\operatorname{Ext}_{R}^{1}(R / I, A)=0$ for all finitely generated left ideals $I$ of $R$.

We have been able to prove the converse of the preceding corollary only under the further assumption that $R$ is semihereditary (see Theorem 4 below).

Corollary 2. An R-module $A$ is absolutely pure if and only if for every diagram

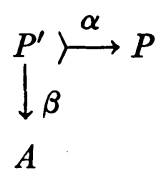

with $P^{\prime}$ finitely generated and $P$ projective there exists a homomorphism $\gamma: P \rightarrow A$ such that $\gamma \alpha=\beta$.

Proof. Clearly we may assume that $P^{\prime}$ is a submodule of $P$ and that $P$ is actually free. Since $P^{\prime}$ is finitely generated, there is then a finitely generated free direct summand $F$ of $P$ such that $P^{\prime} \subseteq F$. Therefore finding the desired $\gamma$ is equivalent to extending $B$ to $F$. But from the exact sequence

$$
\operatorname{Hom}_{R}(F, A) \rightarrow \operatorname{Hom}_{R}\left(P^{\prime}, A\right) \rightarrow \operatorname{Ext}_{R}^{1}\left(F / P^{\prime}, A\right) \rightarrow 0
$$

that is equivalent to $\operatorname{Ext}_{R}^{1}\left(F / P^{\prime}, A\right)=0$.

We can now give an interesting interpretation of the above characterizations in terms of linear equations. Consider the following finite system of linear equations over the $R$-module $A$

$$
\sum_{j=1}^{n} r_{i j} x_{j}=a_{i} \quad\left(r_{i j} \in R ; a_{i} \in A ; i=1, \cdots, m\right) .
$$

Intuitively, this system is consistent if one can multiply the equations by arbitrary elements of $R$ and add the resulting equations together without obtaining an invalid relation among the $a_{i}$ 's. This can however be formulated more rigorously. View the $x_{i}$ 's as free generators of a free $R$-module $F$ and, for each $i$, let $m_{i}=\sum_{j=1}^{n} r_{i j} x_{j} \in F$. Then the consistency of our system of equations reduces to the correspondence $m_{i} \mapsto a_{i}$ inducing a homomorphism $\phi: M \rightarrow A$ when $M$ is the submodule of $F$ generated by the $m_{i}$ 's. On the other hand, it is clear that 
solving our system of equations is then equivalent to finding a homomorphism $\psi: F \rightarrow A$ that extends $\phi$. For a fuller discussion see [2].

THEOREM 1. An $R$-module $A$ is absolutely pure if and only if every consistent finite system of linear equations over $A$ has a solution in $A$.

The reader is reminded that one characterization (see [3]) of injective modules is that every consistent system of linear equations (with no cardinality restriction on the number of equations or unknowns) be solvable. Theorem 1 is thus one justification for calling absolutely pure modules finitely injective. Another is the fact that absolutely pure modules play precisely the same role relative to semihereditary rings that injectives play relative to hereditary rings. Indeed we have the following self-dual improvement of the familiar Proposition 6.2 in Chapter I of [1].

THEOREM 2. For a ring $R$ the following conditions are equivalent:

(a) $R$ is semihereditary.

(b) Each finitely generated submodule of a projective $R$-module is projective.

(c) The homomorphic image of an absolutely pure $R$-module is absolutely pure.

Proof. The equivalence of (a) and (b) is established in [1]. Consider a diagram

$$
\begin{aligned}
& P \stackrel{\alpha}{\leftarrow} P^{\prime} \\
& Q \stackrel{\beta}{\rightarrow} d^{d_{s} f}
\end{aligned}
$$

where $P$ is projective, $P^{\prime}$ is finitely generated and $Q$ is absolutely pure. Assume (b) , then $P^{\prime}$ is projective and there is a homomorphism $\gamma: P^{\prime} \rightarrow Q$ such that $\beta \gamma=f$. Since $Q$ is absolutely pure, there is a homomorphism $\delta: P \rightarrow Q$ such that $\delta \alpha=\gamma$. But then $(\beta \delta) \alpha=f$ and $Q^{\prime}$ is absolutely pure by Corollary 2. Conversely, assume (c) and now take $Q$ to be injective. Then $Q^{\prime}$ is absolutely pure and we have $\gamma: P \rightarrow Q^{\prime}$ such that $\gamma \alpha=f$. Since $P$ is projective, there is a homomorphism $\delta: P \rightarrow Q$ such that $\beta \delta=\gamma$. Then $\beta(\delta \alpha)=f$ and $P^{\prime}$ is projective by Proposition 5.1 in Chapter I of [1].

A portion of our next proposition is also proved in [4].

Proposition 2. Let $\left\{A_{i}, \phi_{i j}\right\}$ be a direct system of $R$-modules where each $A_{i}$ is absolutely pure. If either (1) $R$ is semihereditary or (2) each $\phi_{i j}$ is a monomorphism, then $A=\operatorname{inj} \lim A_{i}$ is absolutely pure. 
Proof. For each $i$, let $\phi_{i}: A_{i} \rightarrow A$ be the canonical map and consider a homomorphism $f: P^{\prime} \rightarrow A$ where $P^{\prime}$ is a finitely generated submodule of the projective $R$-module $P$. Since $P^{\prime}$ is finitely generated, $f\left(P^{\prime}\right)$ $\subseteq \phi_{i}\left(A_{i}\right)$ for some $i$. Now if each $\phi_{i j}$ is monic, then so is each $\phi_{i}$. Thus under either condition (1) or (2), $\phi_{i}\left(A_{i}\right)$ is absolutely pure and there is a homomorphism $g: P \rightarrow \phi_{i}\left(A_{i}\right) \subseteq A$ extending $f$.

Proposition 2 has several consequences : (i) an arbitrary direct sum of absolutely pure $R$-modules is absolutely pure; (ii) the union of a direct family of absolutely pure submodules is an absolutely pure submodule and consequently (iii) every $R$-module contains a maximal absolutely pure submodule. By Theorem 2 and Proposition 2 it is then clear that for modules over a semihereditary ring the sum of an arbitrary family of absolutely pure submodules is absolutely pure. Thus if $R$ is semihereditary, then every $R$-module contains a unique maximal absolutely pure submodule. It is well known that a ring $R$ is Noetherian if and only if an arbitrary direct sum of injective $R$ modules is injective. Thus if $R$ is not Noetherian, we can form a direct sum of injective $R$-modules which is not injective but which is necessarily absolutely pure. This observation and Corollary 1 together yield

TheOREM 3. A ring $R$ is Noetherian if and only if every absolutely pure $R$-module is injective.

We next prove a restricted converse of Corollary 1.

THEOREM 4. If $R$ is semihereditary, then an $R$-module $A$ is absolutely pure if and only if $\operatorname{Ext}_{R}^{1}(R / I, A)=0$ for all finitely generated left ideals $I$ of $R$.

Proof. Assume that $\operatorname{Ext}_{R}^{1}(R / I, A)=0$ for all finitely generated $I$. Consider an arbitary homomorphism $f: M \rightarrow A$ where $M$ is a finitely generated submodule of a finitely generated free $R$-module $F$. The proof is by induction on the number of generators of $F$. If $F \neq 0$, we can write $F=R x \oplus V$ where $V$ is free with fewer generators than $F$. Let $I=\{r \in R: r x \in M+V\}$. Then there is an obvious isomorphism $I \simeq M / M \cap V$. Therefore $I$ is finitely generated and, since $R$ is semihereditary, projective. Thus $M \cap V$ is a direct summand of $M$ and consequently finitely generated. By induction, there is a homomorphism $\xi: V \rightarrow A$ such that $\xi|M \cap V=f| M \cap V$ and hence a unique homomorphism $\theta: M+V \rightarrow A$ extending both $f$ and $\xi$. Define $\phi: I \rightarrow A$ by $\phi(r)=\theta(r x)$ for all $r \in R$. Since $\operatorname{Ext}_{R}^{1}(R / I, A)=0$, there is a homomorphism $\psi: R \rightarrow A$ that extends $\phi$. The mapping $g: F \rightarrow A$ defined by $g(v+r x)=\xi(v)+\psi(r)$ for all $v \in V$ and $r \in R$ is a homomorphism extending $f$. 
Remark. As we only used the hypothesis that $R$ was semihereditary to show that $M \cap V$ was finitely generated, our proof of Theorem 4 applies to the more general class of coherent rings.

Regular rings (in the sense of von Neumann) also admit a characterization in terms of absolutely pure modules.

THEOREM 5. A ring $R$ is regular if and only if every $R$-module is absolutely pure.

PRoof. Let $R$ be a regular ring. Then every finitely generated left ideal $I$ of $R$ is of the form $R e$ with $e$ idempotent and is consequently a direct summand of $R$. Thus both $I$ and $R / I$ are projective $R$-modules. Therefore $R$ is semihereditary and $\operatorname{Ext}_{R}^{1}(R / I, A)=0$ for all $R$-modules $A$ and all finitely generated left ideals $I$.

Conversely, assume that all $R$-modules are absolutely pure and let $I$ be a finitely generated left ideal of $R$. Then $I$ is absolutely pure as an $R$-module and consequently $\operatorname{Ext}_{R}^{1}(R / I, I)=0$. Therefore $I$ is a direct summand of $R$ and hence $I=R e$ for some idempotent $e$; that is, $R$ is regular.

Combining Theorems 3 and 5 , we have the familiar fact that every $R$-module is injective if and only if $R$ is semisimple (in the classical sense).

If $A$ is an absolutely pure $R$-module it is easy to see that $A$ is divisible, that is, $r A=A$ for all nonzero-divisors $r \in R$. Indeed, for any nonzero-divisor $r \in R$ and $x \in A$, consider the homomorphism $\gamma: R r$ $\rightarrow A$ mapping $r$ to $x$. Since $A$ is absolutely pure, there is an extension $\psi: R \rightarrow A$. Then $x=r \psi(1) \in r A$.

THEOREM 6. Let $R$ be a commutative integral domain. Then $R$ is a Priifer ring if and only if every divisible $R$-module is absolutely pure.

Proof. Let $R$ be a Prüfer ring, that is, $R$ is a semihereditary commutative integral domain. By [1, Chapter VII, Proposition 3.4], $\operatorname{Ext}_{R}^{\mathrm{t}}(R / I, A)=0$ whenever $I$ is finitely generated and $A$ is divisible, that is, every divisible $R$-module is absolutely pure by Theorem 4 . Conversely, if every divisible pure $R$-module is absolutely pure, then $R$ is semihereditary by Theorem 2 .

For Prüfer domains we have two further interesting theorems.

Theorem 7. Let $R$ be a Prüfer ring. If $A$ is a divisible $R$-module and $M$ is a countably generated torsion-free $R$-module, then $\operatorname{Ext}_{R}^{1}(M, A)=0$.

Proof. Let $A$ be a submodule of $K$ with $K / A \cong M$. Since $M$ is countably generated, $K$ is the union of an ascending sequence of submodules $K_{0}=A \subseteq K_{1} \subseteq \cdots \subseteq K_{n} \subseteq \cdots$ with $K_{n} / A$ finitely generated. But finitely generated torsion-free modules over a Prüfer ring 
are necessarily projective by [1, Chapter VII, Proposition 4.1] and are therefore finitely presented. Thus each $K_{n} / A$ is finitely presented and consequently $K_{n+1} / K_{n}$ is finitely presented for each $n$. To show that $A$ is a direct summand of $K$ it suffices to construct a sequence of homomorphisms $\phi_{n}: K_{n} \rightarrow A$ such that $\phi_{0}=1_{A}$ and $\phi_{n+1} \mid K_{n}=\phi_{n}$ for all $n$. That such a construction can be accomplished inductively follows immediately from the observation that $\operatorname{Ext}_{R}^{1}\left(K_{n+1} / K_{n}, A\right)=0$ for all $n$.

That the countability hypothesis in Theorem 7 cannot be removed is a consequence of remarks following Theorem 1.2 in [5].

TheOREM 8. Let $R$ be a Prüfer domain with quotient field $Q$. If every $R$-submodule of $Q$ is countably generated, then $\operatorname{Ext}_{R}^{1}(M, A)=0$ whenever $M$ is torsion-free and $A$ is divisible.

Proof. Since $M$ is torsion-free, it is not difficult to see that $M$ is the union of a well-ordered family $\left\{M_{\alpha}\right\}_{\alpha<\lambda}$ of submodules such that (1) $M_{\alpha} \subseteq M_{\beta}$ for $\alpha<\beta$, (2) $M_{\alpha}=\mathrm{U}_{\beta<\alpha} M_{\beta}$ if $\alpha$ is a limit ordinal and (3) $M_{\alpha+1} / M_{\alpha}$ is isomorphic to an $R$-submodule of $Q$ for all $\alpha$. But then our hypothesis on $Q$ and Theorem 7 yield $\operatorname{Ext}_{R}^{1}\left(M_{\alpha+1} / M_{\alpha}, A\right)=0$. The proof is then completed by a transfinite induction similar to the finite induction in the proof of Theorem 7. Note that condition (2) insures that no difficulties arise at limit ordinals.

Corollary 3. Let $R$ and $Q$ be as in Theorem 8. Then $h d_{R} M \leqq 1$ for every torsion free $R$-module $M$ and $\mathrm{gl} . \operatorname{dim} . R \leqq 2$.

Proof. Let $D$ be an injective envelope of the $R$-module $N$. Then $\operatorname{Ext}_{R}^{2}(M, N) \simeq \operatorname{Ext}_{R}^{1}(M, D / N)=0$ if $M$ is torsion-free. Now let $M$ and $N$ be arbitrary $R$-modules and consider a short exact sequence $K\lrcorner F \rightarrow M$ with $F$ free. Then $\operatorname{Ext}_{R}^{3}(M, N) \simeq \operatorname{Ext}_{R}^{2}(K, N)=0$ since $K$ is torsion-free.

\section{REFERENCES}

1. H. Cartan and S. Eilenberg, Homological algebra, Princeton Univ. Press, Princeton, N. J., 1956. MR 17, 1040.

2. A. Kertesz, The general theory of linear equation systems over semisimple rings, Publ. Math. Debrecen 4 (1955), 79-86. MR 16, 991.

3. - Systems of equations over modules, Acta Sci. Math. (Szeged) 18 (1957), 207-234. MR 19, 1155.

4. B. Maddox, Absolutely pure modules, Proc. Amer. Math. Soc. 18 (1967), 155158. MR 37 \#248.

5. E. Matlis, Divisible modules, Proc. Amer. Math. Soc. 11 (1960), 385-391. MR $22 \# 6839$.

Vanderbilt University, Nashville, Tennessee 37203 